\title{
Quasipolynomial Approach to Simultaneous Robust Control of Time-Delay Systems
}

\author{
Nikolaj Semenič, ${ }^{1}$ Andrej Sarjaš, ${ }^{2}$ Amor Chowdhury, ${ }^{1}$ and Rajko Svečko ${ }^{2}$ \\ ${ }^{1}$ Margento R\&D, Gosposvetska c. 84, 2000 Maribor, Slovenia \\ ${ }^{2}$ Faculty of Electrical Engineering and Computer Science, University of Maribor, Smetanova Ulica 17, 2000 Maribor, Slovenia \\ Correspondence should be addressed to Nikolaj Semenič; semenic@gmail.com
}

Received 6 June 2014; Revised 12 August 2014; Accepted 17 August 2014; Published 11 September 2014

Academic Editor: Zhan Shu

Copyright (C) 2014 Nikolaj Semenič et al. This is an open access article distributed under the Creative Commons Attribution License, which permits unrestricted use, distribution, and reproduction in any medium, provided the original work is properly cited.

\begin{abstract}
A control law for retarded time-delay systems is considered, concerning infinite closed-loop spectrum assignment. An algebraic method for spectrum assignment is presented with a unique optimization algorithm for minimization of spectral abscissa and effective shaping of the chains of infinitely many closed-loop poles. Uncertainty of plant delays of a certain structure is considered in a sense of a robust simultaneous stabilization. Robust performance is achieved using mixed sensitivity design, which is incorporated into the addressed control law.
\end{abstract}

\section{Introduction}

Time-delay systems are an important and well established topic in modern control theory [1-3]. Its diverse applications, for instance, in dynamics of fluids, internal combustion, heating systems, regenerative chatter in metal cutting, and networked control systems, led to the development of various complex approaches to system and controller synthesis [4-8]. Infinite dimensional spectrum of such systems might cause difficulties in appropriate spectrum assignment using standard control laws, which consequently means that stabilization cannot be always achieved. Due to the complexity of the spectrum, much interest has been shown in the development of control laws based on the computation of rightmost poles, since such algorithms ensure results with high precision [9-12]. In principle, with a limited number of controller parameters only a few poles can be placed to desired positions in the complex plane [13]. As it has been shown in [8], some controller parameters might be used to directly assign a few dominant poles to an arbitrary position in the complex plane. The remaining controller parameters are then used to shift the chains of infinitely many system poles as far to the left of the dominant poles as possible. Another pole-placement-based technique has been introduced in [14] for retarded systems and in [15] for neutral systems. The method is based on continuous modifications applied to controller parameters in order to shift the rightmost or unstable poles to the left half plane in a quasicontinuous way, while monitoring other eigenvalues. A direct optimization approach $[16,17]$, used to minimize the spectral abscissa, has been introduced in [18].

Alternative eigenvalue based control laws might be considered, which incorporate different algebraic approaches [19]. General SISO time-delay plants treated in the form of a transfer function and its admissible coprime-inner/outer factorizations, derived by using numerical algorithms for computation of closed-loop poles, were discussed in [20]. In addition, an optimal $\mathscr{H}_{\infty}$ controller was designed.

In this paper, a control law for retarded time-delay systems is considered, which results in a controller in the form of a transfer function as a quotient of quasipolynomials. An individual quasipolynomial in the numerator and the denominator consists of several delayed terms that result in a closedloop quasipolynomial of a certain structure of sequentially solvable polynomial Diophantine equations. The structure of the controller is examined and derived in the case of infinite solutions of individual polynomial equations. As has been 
shown in [21] only a few such equations can be solved for an arbitrary polynomial, which suggests that the remainder of the closed-loop spectrum cannot be assigned accordingly. In [21], the unsuitable dynamics of the remaining spectrum has been algebraically eliminated by the specific structure imposed on the controller, which allows finite spectrum assignment. In our presented work, elimination of any part of the spectrum is not admissible and therefore we tackle the problem of infinite closed-loop spectrum assignment, which is indispensable especially in the case of uncertain delays. As soon as delays are perturbed, any delay compensation technique based on preestimated delays does not eliminate delayed terms out of the closed-loop completely, which results in an infinite closed-loop spectrum. We demonstrate that certain estimation technique of a predetermined region of poles [22] might be used in order to shape infinite closed-loop spectrum as well as infinite closed-loop spectrum with uncertain delays. We present an algebraic method for infinite closed-loop spectrum assignment, which reduces the number of parameters in the search routine for the appropriate stabile closed-loop spectrum. The algorithm for the search routine of the appropriately shaped infinite closedloop spectrum is presented. It is shown that mixed sensitivity design might be incorporated into the addressed control law regarding uncertain time delays to obtain an optimal controller.

The paper is organized as follows. After some preliminaries, we derive different controller parameterizations concerning different types of free polynomials. Then, we propose an algebraic method for closed-loop spectrum assignment and present a spectrum shaping technique, where we show spectrum shaping with uncertain delays as well. After that, we give algorithm of a search routine for appropriate stabile closedloop spectrum and present the mixed sensitivity design for robust controller synthesis. Finally, we demonstrate our results on an example and give final remarks in conclusions.

\section{Preliminaries}

Time-delay system of a retarded type, with internal or state and output discrete commensurate delays, is defined as in $[22,23]:$

$$
\begin{aligned}
& \dot{x}(t)=\bar{A}_{0} x(t)+\sum_{i=1}^{\bar{q}} \bar{A}_{i} x\left(t-h_{i} \tau\right)+\bar{B}_{0} u(t), \\
& y(t)=\bar{C}_{0} x(t)+\sum_{i=1}^{\bar{q}} \bar{C}_{i} x\left(t-h_{i} \tau\right)+\bar{D}_{0} u(t),
\end{aligned}
$$

where $\bar{A}_{i} \in \mathbb{R}^{n \times n}, \bar{B}, \bar{C}_{i} \in \mathbb{R}^{n}, i \in \mathbb{N}_{1}^{\bar{q}}$, are system defined parameters, $b(t), y(t) \in \mathbb{R}$ are input and output scalars, $x(t) \in \mathbb{R}^{n}$ is state vector, time delays $\left\{h_{1} \tau<\cdots<h_{i} \tau<\right.$ $\left.\cdots<h_{\bar{q}} \tau\right\}$ are expressed as multiples of $\tau \in \mathbb{R}_{+}, h_{i} \in \mathbb{N}$, and initial condition is a segment of continuous functions $C\left(\left[-h_{i} \tau, 0\right], \mathbb{R}^{n}\right)$ equipped with a supremum norm.
Definition 1. Let $Q_{i}(s)$ for $i \in \mathbb{Z}_{0}^{q}$ be polynomials with real coefficients and $d_{i}$ are nonnegative real numbers in an ascending order. A function of the form

$$
Q(s)=\sum_{i=0}^{q} Q_{i}(s) e^{-d_{i} s}
$$

is a retarded quasipolynomial if $\operatorname{deg} Q_{0}>\operatorname{deg} Q_{i}, i \in \mathbb{N}_{1}^{q}$.

System (1) can be represented with a SISO transfer function $[19,21]$ :

$$
\begin{aligned}
G(s) & =\frac{B(s)}{A(s)} \\
& =\sum_{i=0}^{\bar{q}} \bar{C}_{i}^{T} e^{-h_{i} \tau s}\left(s I-\sum_{i=0}^{\bar{q}} \bar{A}_{i} e^{-h_{i} \tau s}\right)^{-1} \bar{B}_{0}+\bar{D}_{0} u,
\end{aligned}
$$

where $A(s)$ and $B(s)$ are retarded quasipolynomials:

$$
\begin{aligned}
A(s) & =\operatorname{det}\left(s I-\sum_{i=0}^{\bar{q}} \bar{A}_{i} e^{-h_{i} \tau s}\right)=\sum_{i=0}^{n_{h}} A_{i}(s) e^{-i \tau s} \\
& =\sum_{j=0}^{n} A_{j}^{*}\left(e^{-\tau s}\right) s^{j}=\sum_{i=0}^{n_{h}} \sum_{j=0}^{n} a_{i j} s^{j} e^{-i \tau s},
\end{aligned}
$$

with $i \in \mathbb{Z}_{0}^{n_{h}}$ and $j \in \mathbb{Z}_{0}^{n}$,

$$
\begin{aligned}
B(s) & =\sum_{i=0}^{\bar{q}} \bar{C}_{i}^{T} e^{-h_{i} \tau s} \operatorname{Adj}\left(s I-\sum_{i=0}^{\bar{q}} \bar{A}_{i} e^{-h_{i} \tau s}\right) \bar{B}_{0}+\bar{D}_{0} A(s) \\
& =\sum_{i=0}^{m_{h}} B_{i}(s) e^{-i \tau s}=\sum_{j=0}^{m} B_{j}^{*}\left(e^{-\tau s}\right) s^{j}=\sum_{i=0}^{m_{h}} \sum_{j=0}^{m} b_{i j} s^{j} e^{-i \tau s},
\end{aligned}
$$

with $i \in \mathbb{Z}_{0}^{m_{h}}$ and $j \in \mathbb{Z}_{0}^{m} ; A^{*}\left(e^{-\tau s}\right)=\sum_{i=0}^{n_{h}} a_{i j} s^{j} e^{-i \tau s}$ and $B^{*}\left(e^{-\tau s}\right)=\sum_{i=0}^{m_{h}} b_{i j} s^{j} e^{-i \tau s}$ are alternative polynomials in variable $e^{-\tau s}$ and represent an equivalent description of quasipolynomials as Definition 1.

Considering control law

$$
u(t)=\frac{P(s)}{R(s)}(y(t)-r(t)),
$$

with $r(t)$ being any given bounded reference signal, $P(s)$ and $R(s)$ are retarded quasipolynomials of the form (3) and the controller structure

$$
\begin{aligned}
K(s) & =\frac{P(s)}{R(s)}=\frac{\sum_{i=0}^{l_{h}} P_{i}(s) e^{-i \tau s}}{\sum_{i=0}^{k_{h}} R_{i}(s) e^{-i \tau s}} \\
& =\frac{\sum_{j=0}^{l} P_{j}^{*}\left(e^{-\tau s}\right) s^{j}}{\sum_{j=0}^{k} R_{j}^{*}\left(e^{-\tau s}\right) s^{j}} \\
& =\frac{\sum_{i=0}^{l_{h}} \sum_{j=0}^{l} p_{i k} s^{j} e^{-i \tau s}}{\sum_{i=0}^{k_{h}} \sum_{j=0}^{k} r_{i k} s^{j} e^{-i \tau s}} .
\end{aligned}
$$


Assumption 1. We consider controller structure (8) with the same number of delays in the numerator and the denominator $k_{h}=l_{h}=v$ and $\operatorname{deg} R_{0}=\operatorname{deg} P_{0}>\operatorname{deg} R_{i}=\operatorname{deg} P_{i}$, $i \in \mathbb{N}_{1}^{v}$, with $R_{0}(s)$ being monic.

Assumption 2. Quasipolynomials $A(s)$ and $B(s)$ as well as polynomials $A_{0}(s)$ and $B_{0}(s)$ have no common zeroes. Complex Laplace argument $(s)$ is hereafter omitted for clarity.

Controller (8) results in closed-loop characteristic quasipolynomial of the form

$$
\begin{aligned}
D & =\sum_{i=0}^{v+q} D_{i} e^{-i \tau s}=A R+B P \\
& =\sum_{i=0}^{v+q} \sum_{j=\operatorname{Max}(0, i-v)}^{\operatorname{Min}(i, q)}\left(A_{j} R_{i-j}+B_{j} P_{i-j}\right) e^{-i \tau s},
\end{aligned}
$$

where $q=\max \left(n_{h}, m_{h}\right)$ and $v$ is as Assumption 1 .

Regarding the closed-loop structure (9), which might be represented as a sum of several polynomial Diophantine equations with specific delay terms $e^{-i \tau s}$, the following result can be stated.

Proposition 2. Let $A, B, P$, and $R$ be quasipolynomials given by Definition 1 and Assumptions 1 and 2 hold. Then individual polynomial Diophantine equations in (9) for $i \in \mathbb{Z}_{0}^{v}$,

$$
A_{0} R_{i}+B_{0} P_{i}=D_{i}-\sum_{j=1}^{i}\left(A_{j} R_{i-j}+B_{j} P_{i-j}\right)
$$

have solutions for arbitrary $D_{i}$, where the equation for $i=0$ has always infinite solutions ( $\left.\operatorname{deg} R_{0}>\operatorname{deg} A_{0}-1\right)$ and equations for $i \in \mathbb{N}_{1}^{v}$ have a unique $\left(\operatorname{deg} R_{i}=\operatorname{deg} A_{0}-1\right)$ or infinite solutions ( $\operatorname{deg} R_{i}>\operatorname{deg} A_{0}-1$ ), if the following is true:

$$
\begin{aligned}
\operatorname{deg} R_{0}= & \operatorname{deg} P_{0} \leq \operatorname{deg} R_{i}+\operatorname{deg} A_{0} \\
& -\max \left(\max _{j}\left(\operatorname{deg} A_{j}\right), \max _{k}\left(\operatorname{deg} B_{k}\right)\right),
\end{aligned}
$$

$j \in \mathbb{N}_{1}^{n_{h}}, k \in \mathbb{N}_{1}^{m_{h}}$, and $d_{i \operatorname{deg} D_{i}} \neq a_{i \operatorname{deg} A_{i}}+b_{i \operatorname{deg} B_{i}} p_{0 \operatorname{deg} P_{0}}$ incase of $\operatorname{deg} A_{0}+\operatorname{deg} R_{i}=\operatorname{deg} A_{i}+\operatorname{deg} R_{0}=\operatorname{deg} B_{i}+\operatorname{deg} P_{0}, i \in \mathbb{N}_{1}^{v}$.

Proof. Polynomial equations (10) need to be solved sequentially since individual controller variables are present in several equations. Equations (10) for $i \in \mathbb{N}_{1}^{v}$ become

$$
A_{0} R_{i}+B_{0} P_{i}=D_{i}-\cdots-A_{i} R_{0}-B_{i} P_{0}
$$
hold:

For the expression on the left side, the following must

$$
\operatorname{deg} R_{i} \geq \operatorname{deg} A_{0}-1
$$

in order, (12) remains consistent polynomial equation. For the same reason, the degree of the expression on the right must not exceed the degree of the expression on the left; therefore, the following must hold:

$$
\begin{aligned}
\max & \left(\operatorname{deg} A_{i}, \operatorname{deg} B_{i}\right)+\operatorname{deg} R_{0} \\
& \leq \operatorname{deg} A_{0}+\operatorname{deg} R_{i}=\operatorname{deg} D_{i} .
\end{aligned}
$$

From (14), (11) follows straightforwardly.

In the case of the equality in expression (14), selecting leading term of $D_{i}$ according to $d_{i \operatorname{deg} D_{i}}=a_{i \operatorname{deg} A_{i}}+$ $b_{i \operatorname{deg} A_{i}} p_{0 \operatorname{deg} P_{0}}$ results in the reduction of the degree of the expression on the right and therefore inconsistency of (12).

It can be shown that the first equation of (10) for $i=0$ has always infinite solutions. The minimal allowed degree of $R_{i}$ is $\operatorname{deg} R_{i}=\operatorname{deg} A_{0}-1, i \in \mathbb{N}_{1}^{v}$, and the maximal allowed degree of $R_{0}$ is determined by the equality of (11). If we join the two expressions, we can conclude that

$$
\begin{aligned}
\operatorname{deg} R_{0}= & 2 \operatorname{deg} A_{0}-\max \left(\max _{j}\left(\operatorname{deg} A_{j}\right), \max _{k}\left(\operatorname{deg} B_{k}\right)\right) \\
& -1>\operatorname{deg} A_{0}-1,
\end{aligned}
$$

and consequently the first Diophantine equation always has infinite solutions.

Remark 3. According to Proposition 2, only $v$ out of $v+q$ equations can be arbitrarily assigned as equations for $i \in \mathbb{N}_{v+1}^{v+q}$ consist of controller variables already assigned in equations $i \in \mathbb{Z}_{0}^{v}$, which suggests that infinite closed-loop spectrum assignment is not a straightforward task.

\section{Controller Parameterization}

For the parameterization of infinite number of controllers, we make the following assumption and give the result.

Assumption 3. To derive free parameters in all solvable Diophantine equations (10), the following must hold: $\operatorname{deg} R_{i}>$ $\operatorname{deg} A_{0}-1$ and $\operatorname{deg} R_{0}=2 \operatorname{deg} A_{0}-\max _{j}\left(\operatorname{deg} A_{j}\right)>\operatorname{deg} A_{0}$, which we derive with the same procedure as (15) in the proof of Proposition 2.

Theorem 4. Considering Assumptions 1, 2, and 3, the controller (8) can be expressed in the form

$$
K_{\kappa}=\left\{\frac{\sum_{i=0}^{v} P_{i} e^{-i \tau s}+A_{0} \sum_{i=0}^{v} \kappa_{i} e^{-i \tau s}+A_{1} e^{-\tau s} \sum_{i=0}^{\nu-1} \kappa_{i} e^{-i \tau s}+\cdots+A_{n_{h}} e^{-n_{h} \tau s} \sum_{i=0}^{v-n_{h}} \kappa_{i} e^{-i \tau s}}{\sum_{i=0}^{v} R_{i} e^{-i \tau s}-B_{0} \sum_{i=0}^{v} \kappa_{i} e^{-i \tau s}-B_{1} e^{-\tau s} \sum_{i=0}^{v-1} \kappa_{i} e^{-i \tau s}-\cdots-B_{m_{h}} e^{-m_{h} \tau s} \sum_{i=0}^{v-m_{h}} \kappa_{i} e^{-i \tau s}}=\frac{P+\sum_{i=0}^{v} \sum_{k=0}^{\min \left(i, n_{h}\right)} A_{k} \kappa_{i-k} e^{-i \tau s}}{R-\sum_{i=0}^{v} \sum_{k=0}^{\min \left(i, m_{h}\right)} B_{k} \kappa_{i-k} e^{-i \tau s}}\right\},
$$


where $\kappa_{i}$ are polynomials of degree $0 \leq \operatorname{deg} \kappa_{i}=\operatorname{deg} R_{i}-$ $\operatorname{deg} A_{0}, i \in \mathbb{Z}_{0}^{v}$, which results in the following closed-loop characteristic quasipolynomial:

$$
\begin{aligned}
& D= A\left(R-\sum_{i=0}^{v} \sum_{k=0}^{\min \left(i, m_{h}\right)} B_{k} \kappa_{i-k} e^{-i \tau s}\right) \\
&+B\left(P+\sum_{i=0}^{v} \sum_{k=0}^{\min \left(i, n_{h}\right)} A_{k} \kappa_{i-k} e^{-i \tau s}\right) \\
&= \sum_{i=0}^{v} \sum_{j=0}^{\min (i, q)}\left(A_{j} R_{i-j}+B_{j} P_{i-j}\right) e^{-i \tau s} \\
&+\sum_{i=v+1}^{v+q} \sum_{j=i-v}^{\min (i, q)}\left(A_{j}\left(R_{i-j}-\sum_{k=0}^{\min \left(i-j, m_{h}\right)} B_{k} \kappa_{i-j-k}\right)\right. \\
&\left.+B_{j}\left(P_{i-j}+\sum_{k=0}^{\min \left(i-j, n_{h}\right)} A_{k} \kappa_{i-j-k}\right)\right) e^{-i \tau s} .
\end{aligned}
$$

The proof of Theorem 4 can be found in the appendix.

Remark 5. Polynomial equations (10) need to be solved sequentially for each change of a specific free parameter in an individual polynomial equation. The importance of the result of Theorem 4 lies in the fact that all the infinitely many solutions of individual polynomial equations are expressed using free polynomials $\kappa_{i}$. It is evident that as soon as controller variables $P$ and $R$ are derived it is not needed to solve polynomial equations again in case of a change of any parameter of free polynomials.

In the same way as individual Diophantine equations, the whole closed-loop characteristic quasipolynomial (9) can be treated as a single Diophantine equation. This leads to the following closed-loop [24] representation:

$$
\begin{aligned}
D & =A(R-B \lambda)+B(P+A \lambda) \\
& =A R+B P,
\end{aligned}
$$

where $\lambda$ is a sum of polynomials $\lambda_{i}$ as defined in (19) and a corresponding controller structure of the form

$$
\begin{array}{r}
K_{\lambda}=\left\{\frac{P+A \lambda}{R-B \lambda}\right\}=\left\{\frac{\sum_{i=0}^{v} P_{i} e^{-i \tau s}+\sum_{i=0}^{n_{h}} A_{i} e^{-i \tau s} \sum_{i=0}^{v} \lambda_{i} e^{-i \tau s}}{\sum_{i=0}^{v} R_{i} e^{-i \tau s}-\sum_{i=0}^{m_{h}} B_{i} e^{-i \tau s} \sum_{i=0}^{v} \lambda_{i} e^{-i \tau s}}\right. \\
\left.=\frac{P+\sum_{i=0}^{v+n_{h}} \sum_{k=0}^{\min \left(i, n_{h}\right)} A_{k} \lambda_{i-k} e^{-i \tau s}}{R-\sum_{i=0}^{v+m_{h}} \sum_{k=0}^{\min \left(i, m_{h}\right)} B_{k} \lambda_{i-k} e^{-i \tau s}}\right\},
\end{array}
$$

where $\lambda_{i}$ are polynomials of degree $0 \leq \operatorname{deg} R_{i}-\operatorname{deg} A_{0} \leq$ $\operatorname{deg} \lambda_{i} \leq \operatorname{deg} R_{i}-\operatorname{deg} B_{i}-1, i \in \mathbb{Z}_{0}^{v}$.

The two presented controller structures (16) and (19) consisting of different types of free polynomials might be joined in a unified controller structure $K_{\gamma}$.

Theorem 6. Considering Assumptions 1, 2, and 3, unified controller structure

$$
K_{\gamma}=\left\{\frac{P+\sum_{i=0}^{v} \sum_{k=0}^{\min \left(i, n_{h}\right)} A_{k} \kappa_{i-k} e^{-i \tau s}+\sum_{i=0}^{v+n_{h}} \sum_{k=0}^{\min \left(i, n_{h}\right)} A_{k} \lambda_{i-k} e^{-i \tau s}}{R-\sum_{i=0}^{v} \sum_{k=0}^{\min \left(i, m_{h}\right)} B_{k} \kappa_{i-k} e^{-i \tau s}-\sum_{i=0}^{v+m_{h}} \sum_{k=0}^{\min \left(i, m_{h}\right)} B_{k} \lambda_{i-k} e^{-i \tau s}}\right\},
$$

where $\lambda_{i}$ and $\kappa_{i}$ are polynomials of degree as in (16) and (19), respectively, results in the closed-loop characteristic quasipolynomial (17).

The proof of Theorem 6 can be found in the appendix.

\section{Infinite Closed-Loop Spectrum Assignment}

Due to the part of the closed-loop quasipolynomial that cannot be assigned arbitrarily, finite closed-loop spectrum assignment cannot be achieved without the cancelation or compensation of that part of the spectrum [21]. On the other hand, appropriate infinite closed-loop spectrum assignment might be achieved with the help of a numerical routine for reliably computing the rightmost poles of a closed-loop spectrum. The quasipolynomial of the form (9) might be determined with a direct search routine by continuously selecting controller variables and by closely observing the rightmost dominant poles of the closed-loop system by shifting the chains of infinitely many poles as far to the left of the dominant poles as possible. This might be achieved by minimizing the norm of the delayed terms in (9) [22]. Such search routine is computationally expensive since the number of controller parameters rapidly increases with the higher order of individual controller polynomials and the number of delayed controller polynomials. Another search routine could be constructed in a manner of continuously selecting closed-loop solution objectives of (9) represented as an overdetermined system of linear equations, where the individual closed-loop solution objective is designed by selecting values of the first equation representing a Hurwitzian polynomial and zeroing all other equations. Such solution objective will tend to minimize the effects of delayed parts of (9) by shifting the chains of infinitely many poles as far to the left as possible and consists of far less search parameters. The control objective can be expressed as an overdetermined system of linear equations by deriving the approximate solution of the expression $\min _{x}\|S x-\bar{D}\|,[25]$ using ordinary least squares method by

$$
x=\left(S^{T} S\right)^{-1} S^{T} \bar{D}
$$


where $x=\left[\begin{array}{lllll}\bar{R}_{0} & \bar{P}_{0} & \cdots & \bar{R}_{v} & \bar{P}_{v}\end{array}\right]^{T}, x \in \mathbb{R}^{\sum_{i=0}^{v} 2 \operatorname{deg} R_{i}}, \bar{R}_{i} \in$ $\mathbb{R}^{\operatorname{deg} R_{i}}$, and $\bar{P}_{i} \in \mathbb{R}^{\operatorname{deg} P_{i}}, i \in \mathbb{Z}_{0}^{v}$, are controller parameters (8), $S$ is Sylvester matrix of (9), and $\bar{D}$ is the appropriately sized solution objective of the closed-loop quasipolynomial.

Through a close inspection of (9), we can conclude that, by selecting the sufficiently large number of delayed terms in (8), the solutions of the first few equations will never be included in the last few equations in (9), which do not have arbitrary solutions. Having that in mind, we can directly assign to the first few equations an arbitrary closed-loop polynomial $D_{i}$ and derive solutions of the remaining equations according to (21), which is presented in the following result defining the sufficient structure of controller (8).

Proposition 7. The solutions of the first jth polynomial equations in the sum (9) or (17) will not be part of the $\mathbb{N}_{v+1}^{v+q}$ equations if $v=q+j, j \in \mathbb{N}$. Therefore, assigning first $j$ th equations arbitrarily and rearranging remaining equations $\mathbb{N}_{j+1}^{v+q}$ in (9) into (21), where the solution objective $\bar{D}$ is appropriately sized vector of zeroes and

$$
x=\left[\begin{array}{lllll}
\bar{R}_{j+1} & \bar{P}_{j+1} & \cdots & \bar{R}_{v} & \bar{P}_{v}
\end{array}\right]^{T}, \quad x \in \mathbb{R}^{\sum_{i=j+1}^{v} 2 \operatorname{deg} R_{i}},
$$

will result in a consistent system of overdetermined equations.

Proof. According to Proposition 2, equations for $i \in \mathbb{Z}_{0}^{v}$ in the sum (9) have arbitrary solutions. Choosing $v=q+j$, the remaining equations, which do not have arbitrary solutions, become for $i \in \mathbb{N}_{q+j+1}^{2 q+j}$ and consist of controller variables $P_{i}$ and $R_{i}$, where $i \in \mathbb{N}_{j+1}^{q+j}$. Therefore, controller variables $P_{i}$ and $R_{i}$, where $i \in \mathbb{Z}_{0}^{j}$, are not present in the remaining equations. So assigning and exchanging these variables with the values everywhere in (9) and rearranging remaining equations of (9) into (21) will result in a consistent system of overdetermined equations.

Remark 8. The result of Proposition 7 can be directly applied onto a search routine by continuously selecting polynomials of the first or of the first few equations in the sum (9), where the first one is always Hurwitzian, deriving solutions of the first few equations by Proposition 2 and deriving solutions of the remaining equations by (21) and by closely inspecting closed-loop poles of (9). Hence, such search routine allows direct assignment of poles of the first nondelayed equation or of the first few equations and tends to minimize the effects of the remaining delayed equations.

\section{Shaping of the Infinite Closed-Loop Spectrum}

In order to appropriately shape the chains of infinitely many poles in the closed loop, by shifting them as far to the left from the dominant rightmost poles as possible, we can either compute sufficiently many rightmost poles, which is computationally expensive, or use the estimation technique of a predetermined region of poles presented in [9]. From the information of the real part of the rightmost pole $r=$ $\max (\Re(s))$ and the distance from the real part of rightmost pole $d$ on the real axes of the complex plane, we can specify a search criterion based on the predetermined region. This means that all the characteristic poles of the closed-loop quasipolynomial in the vertical strip of the complex plain $d<\mathfrak{R}(s) \leq r$ must belong to the predetermined region of a certain size. The estimation of the region with the belonging poles is given with the following result.

Proposition 9 (see [9]). All characteristic roots of (1) satisfy $\mathfrak{R}(s) \geq \xi, \xi \in \mathbb{R}$, belonging to the set

$$
\begin{aligned}
\Omega_{\xi}:= & \left\{\bigcup \sigma\left(\bar{A}_{0}+\sum_{k=1}^{\bar{q}} \bar{A}_{k} z^{h_{k}}\right): z \in \mathbb{C},|z| \leq e^{-\xi \tau}, k \in \mathbb{N}_{1}^{\bar{q}}\right\} \\
& \bigcap\{s \in \mathbb{C}: \Re(s) \geq \xi\} .
\end{aligned}
$$

The boundary of $\Omega_{\xi}$ is included in

$$
\begin{aligned}
& \psi_{\xi}:=\left(\bigcup_{\omega \in[0,2 \pi)} \sigma\left(\bar{A}_{0}+\sum_{k=1}^{\bar{q}} \bar{A}_{k} e^{-\xi \tau h_{k}} e^{j \omega h_{k}}\right)\right) \\
& \bigcap\{s \in \mathbb{C}: \mathfrak{R}(s) \geq \xi\} .
\end{aligned}
$$

For more information and proof see [26, Appendix A.1].

Remark 10. To derive the boundary $\psi_{0}$ of characteristic roots of (1) in the vertical stripe $d<\Re(s) \leq r$, we need to shift the origin of (1) by $-r+d$, which can be done by introducing new variable $s \rightarrow s-r+d$ in the characteristic matrix $\Delta(s)$ :

$$
\Delta(s):=s I-\bar{A}_{0}-\sum_{k=1}^{\bar{q}} \bar{A}_{k} e^{-s \tau h_{k}} .
$$

After shifting the origin, (24) must be executed in $p h_{q}$ grid points over the interval $[0,2 \pi)$, because the function $\omega \rightarrow$ $e^{j \omega h_{q}}$ has period $2 \pi / h_{q}$. The value of the boundary $\psi_{0}$ might present a constraint of the search routine of the closed-loop quasipolynomial.

\section{Shaping of the Infinite Closed-Loop Spectrum in Case of a Delay Mismatch}

The structure of the delay uncertainty is in a form of multiplicative

$$
\begin{aligned}
& \left\{h_{1} \tilde{\tau}<\cdots<h_{i} \tilde{\tau}<\cdots<h_{q} \tilde{\tau}: \tilde{\tau}=\tau(1+\Delta \bar{\tau}),\right. \\
& \quad \bar{\tau} \in \mathbb{R}, \Delta \in[0,1]\}
\end{aligned}
$$

or additive uncertainty

$$
\begin{aligned}
& \left\{h_{1} \tilde{\tau}<\cdots<h_{i} \tilde{\tau}<\cdots<h_{q} \tilde{\tau}: \widetilde{\tau}=\tau+\frac{\Delta \bar{\tau}}{h_{i}},\right. \\
& \bar{\tau} \in \mathbb{R}, \Delta \in[0,1]\},
\end{aligned}
$$


which both introduce slightly more delayed terms in (9) as a result of multiplication between the delayed terms of the plant and the controller. Therefore, the closed-loop spectrum might change significantly as well. The same constraint as in Proposition 9 and Remark 10 might also be applied to the plant with an uncertain delay. It can be shown that, in the search routine, it is sufficient to consider only the boundary $\psi_{\xi, \bar{\tau}}$ as the boundaries $\psi_{\xi, \Delta \bar{\tau}}$ are included inside of $\psi_{\xi, \bar{\tau}}, \psi_{\xi, \Delta \bar{\tau}} \subseteq \psi_{\xi, \bar{\tau}}$. First we give an important property of timedelay systems.

Proposition 11 (see [22]). If $s$ is a characteristic root of the system (1), then it satisfies

$$
|s| \leq\left\|\bar{A}_{0}\right\|_{2}+\sum_{i=1}^{\bar{q}}\left\|\bar{A}_{i}\right\|_{2} e^{-\Re(s) \tau h_{i}} .
$$

Proof. The expression $\Delta(s)=0(25)$ is equivalent to

$$
s \in \sigma\left(\bar{A}_{0}+\sum_{i=1}^{\bar{q}} \bar{A}_{i} e^{-s \tau h_{i}}\right) .
$$

Interpreting the argument of $\sigma(\cdot)$ as a matrix leads to

$$
|s| \leq\left\|\bar{A}_{0}+\sum_{i=1}^{\bar{q}} \bar{A}_{i} e^{-s \tau h_{i}}\right\|_{2},
$$

from which (28) follows straightforwardly.

Using Proposition 11 and the result in Proposition 9, we can derive the following result.

Proposition 12. For any positive $\Delta \in[0,1], \psi_{\xi, \Delta \bar{\tau}} \subseteq \psi_{\xi, \bar{\tau}}$ holds, where in (28) only the delay is perturbed according to (26) or (27).

Proof. Regarding Proposition 11 and the multiplicative uncertainty (26) leads to

$$
\begin{aligned}
|s| & \leq\left\|\bar{D}_{0}\right\|_{2}+\sum_{k=1}^{q}\left\|\bar{D}_{k}\right\|_{2} e^{-\sigma \tau(1+\Delta \bar{\tau}) h_{k}} \\
& \leq\left\|\bar{D}_{0}\right\|_{2}+\sum_{k=1}^{q}\left\|\bar{D}_{k}\right\|_{2} e^{-\sigma \tau(1+\bar{\tau}) h_{k}},
\end{aligned}
$$

and regarding the additive uncertainty (27) leads to

$$
\begin{aligned}
|s| & \leq\left\|\bar{D}_{0}\right\|_{2}+\sum_{k=1}^{q}\left\|\bar{D}_{k}\right\|_{2} e^{-\sigma\left(\tau h_{k}+\Delta \bar{\tau}\right)} \\
& \leq\left\|\bar{D}_{0}\right\|_{2}+\sum_{k=1}^{q}\left\|\bar{D}_{k}\right\|_{2} e^{-\sigma\left(\tau h_{k}+\bar{\tau}\right)},
\end{aligned}
$$

where $\bar{D}_{i}, i \in \mathbb{Z}_{0}^{q}$, is the closed-loop quasipolynomial (9) in matrix form considering the delay uncertainty. Both expressions (31) and (32) are based on a simple comparison of exponential functions; namely, $e^{-\sigma \Delta \bar{\tau}} \leq e^{-\sigma \bar{\tau}}$ and $e^{-\sigma \tau \Delta \bar{\tau} h_{k}} \leq$ $e^{-\sigma \tau \bar{\tau} h_{k}}$, respectively, $\sigma \in \mathbb{R}_{-}$. Since the area that contains all the characteristic roots in specific complex plane $\mathfrak{R}(s)$ of perturbed system is larger, it is sufficient to consider the boundary $\psi_{\xi, \bar{\tau}}$ of the largest set $\Omega_{\xi, \bar{\tau}}$.

Finally, we can present the algorithm for the derivation of the infinite closed-loop spectrum, considering appropriate shaping of the chains of the infinitely many poles and constraints on the delay mismatch. The main objective of the algorithm is the minimization of function:

$$
(K) \longrightarrow \alpha(K)+\psi_{0}(K)+\psi_{0, \bar{\tau}}(K),
$$

where $\alpha$ is spectral abscissa:

$$
\alpha:=\sup \{\mathfrak{R}(s), s \text { is characteristic root }\} .
$$

Besides the spectral abscissa and the criterion based on Proposition 9, we might improve the objective function (34) by additional constraints, for instance, on the position of the dominant rightmost poles in the complex plane in the sense of damping, overshoot, or rise time [27].

As the spectral abscissa is a nonsmooth function, a gradient sampling algorithm presented in $[16,17]$ or differential evolution [28] might be used for the minimization of (34).

The important part of the algorithm is computation of only a few rightmost characteristic roots of (1). There are several numerical solutions [9-12] in the form of algorithms, with a distinct exception [9], which is based on an estimation of all roots in a predetermined region (24). Such an algorithm can be easily adapted in a way of computation of only a few rightmost roots with continuously shifting of the origin of the complex plane, in such a way that the predetermined region of all roots is as small as possible but large enough that it contains only a few rightmost roots (Remark 10). The shifting of the origin might be performed using a bisection algorithm. In this way, only a few rightmost roots are computed, which is much more efficient in the search for the appropriate closedloop quasipolynomial, in contrast to the derivation of large number of roots, which is computationally very expensive.

Algorithm 13. We have the following.

(1) Choose $v=q+j$ as Proposition 7.

(2) Assign a Hurwitzian polynomial to first and any polynomial to the remaining $j$ th equations according to the optimization method and derive a closed-loop spectrum (9) using Proposition 7.

(3) Derive the first few rightmost poles of (9).

(4) Derive $\psi_{0}$ spectrum of (9) using Proposition 9.

(5) Derive the first few rightmost poles of (9) considering delay uncertainty (26) or (27).

(6) Derive $\psi_{0, \bar{\tau}}$ spectrum of (9) using Proposition 9 and considering delay uncertainty (26) or (27).

(7) Compute the objective function (33) and derive improved values from minimization algorithm for step (2).

(8) Continue with step (2) until appropriate closed-loop spectrum is derived. 


\section{Robust Optimization via Mixed Sensitivity Design}

Mixed sensitivity can be presented as an optimization problem of minimizing the $\mathscr{H}_{\infty}$ norm $[24,29]$ of the

$$
\min _{\lambda}\left\|\left[\begin{array}{c}
W_{S}(s) S(s, \lambda) \\
W_{T}(s) T(s, \lambda)
\end{array}\right]\right\|_{\infty} \leq \gamma, \quad \gamma \in \mathbb{R}_{+},
$$

where $W_{S}(s)$ and $W_{T}(s)$ are weighting filters in a form of frequency depended bounds, which characterize robust stability and robust performance; $S(s, \lambda)$ and $T(s, \lambda)$ are sensitivity and complementary sensitivity. Applying (19) to sensitivity and complementary sensitivity leads to

$$
\begin{aligned}
S(s, \lambda) & =\frac{A(R-B \lambda)}{A R+B P} \\
& =\frac{A\left(R-\sum_{i=0}^{v+m_{h}} \sum_{k=0}^{\min \left(i, m_{h}\right)} B_{k} \lambda_{i-k} e^{-i \tau s}\right)}{A R+B P}, \\
T(s, \lambda) & =\frac{B(P+A \lambda)}{A R+B P} \\
& =\frac{B\left(P+\sum_{i=0}^{v+n_{h}} \sum_{k=0}^{\min \left(i, n_{h}\right)} A_{k} \lambda_{i-k} e^{-i \tau s}\right)}{A R+B P} .
\end{aligned}
$$

When considering the delay uncertainty (26) or (27), structures of $S(s, \lambda)$ and $T(s, \lambda)$ are slightly altered. Therefore, the characteristic quasipolynomial depends on polynomials $\lambda_{i}$ as well:

$$
\begin{aligned}
& \widetilde{S}(s, \lambda)=\frac{\widetilde{A}(R-B \lambda)}{\widetilde{A}(R-B \lambda)+\widetilde{B}(P+A \lambda)}, \\
& \widetilde{T}(s, \lambda)=\frac{\widetilde{B}(P+A \lambda)}{\widetilde{A}(R-B \lambda)+\widetilde{B}(P+A \lambda)},
\end{aligned}
$$

where $\widetilde{A}$ and $\widetilde{B}$ are as in (4), only perturbed according to (26) or (27), respectively. By applying the uncertain $\widetilde{S}(s, \lambda)$ and $\widetilde{T}(s, \lambda)$ to the optimization procedure (35), we derive

$$
\min _{\lambda}\left\|\left[\begin{array}{c}
W_{S}(s) S(s, \lambda) \\
W_{T}(s) T(s, \lambda) \\
\widetilde{W}_{S}(s) \widetilde{S}(s, \lambda) \\
\widetilde{W}_{T}(s) \widetilde{T}(s, \lambda)
\end{array}\right]\right\|_{\infty} \leq \gamma, \quad \gamma \in \mathbb{R}_{+},
$$

with $\widetilde{W}_{S}(s)$ and $\widetilde{W}_{T}(s)$ being weighting filters characterizing sensitivity and complementary sensitivity of the plant with uncertain delays.

Optimal controller design might be performed by minimizing (38) and by closely observing right most poles of $\widetilde{T}(s, \lambda)$ in sense or infinite closed-loop spectrum shaping according to Algorithm 13.

\section{Example: Level Control of a Chain of Evaporators}

The dynamics of the level control of a chain of evaporators may be modeled by a delayed first order plus integrator transfer function $[30,31]$ :

$$
F=\frac{-k e^{-\tau s}}{s(T s+1)},
$$

where parameters $\tau=5 s, k=-0.1$, and $T=5 s$. Time delay considered with appropriate uncertainty, for instance, using (26), $\tilde{\tau}=\tau(1+\Delta 2)$. Representing the transport delay as output delay and incorporating direct nondelayed output connections into the model (39) to represent the model in the form of (2), which might be achieved either by the observer $[32,33]$ or even by a classical smith predictor $[31,34]$, lead to the following model representation:

$$
\widetilde{F}=\frac{-k\left(s+1+e^{-\tilde{\tau} s}\right)}{s(T s+1)} .
$$

The following controller was designed

$$
K_{\gamma}=\frac{P_{0}+P_{5} e^{-5 s}+A_{0} \lambda_{0}+A_{0} \lambda_{5} e^{-5 s}}{R_{0}+R_{5} e^{-5 s}-B_{0} \lambda_{0}-B_{5} \lambda_{0} e^{-5 s}-B_{0} \lambda_{5} e^{-5 s}},
$$

where the number of delayed controller polynomials was set according to Proposition 7, $v=q+j=5+0=5$. The degree of individual controller polynomials was set using the result of Proposition 2. Selecting $\operatorname{deg} P_{5}=\operatorname{deg} R_{5}=1$ and $1<\operatorname{deg} P=\operatorname{deg} R_{0}=2 \leq 3$. According to Theorem 6 and (19) $0 \leq \operatorname{deg} \lambda_{0}=0 \leq 1$ and $0 \leq \operatorname{deg} \lambda_{5}=0 \leq 0$. Applying Algorithm 13, where $\psi_{0}$ and $\psi_{0, \bar{\tau}}$ were computed according to Proposition 9 and Remark 10 for $d=0.2$ (Figure 1). What is clear is that the higher the value $d$ is, the larger the region $\psi_{0, \bar{\tau}}$ is. So from the numerical point of view, $d$ should be set small. Optimal $\mathscr{H}_{\infty}$ controller (38) was designed for $W_{S}=$ $\widetilde{W}_{S}=(0.5 s+0.01746) /(s+0.002182)$ and $W_{T}=\widetilde{W}_{T}=$ $(8 s+4.583) /(s+9.165)$ (Figure 4$)$ with the following controller parameters: $p_{02}=1, p_{01}=-1.3884 \cdot 10^{5}, p_{00}=-5.1942 \cdot 10^{5}$, $p_{51}=-0.4432 \cdot 10^{5}, p_{50}=2.3201 \cdot 10^{5}, r_{02}=1, r_{01}=0.012 \cdot 10^{5}$, $r_{00}=3.4481 \cdot 10^{5}, r_{51}=-44.3231, r_{50}=-655.9617, \lambda_{0}=$ -1000 , and $\lambda_{5}=0$. The step response of $\widetilde{T}(s, \lambda)$ is shown in Figure 2 in blue and of $T(s, \lambda)$ in red. Closed-loop poles of $T(s, \lambda)$ are shown in Figure 3 in blue and of $\bar{T}(s, \lambda), \widetilde{\tau}=3 \tau$, and $\Delta=1$ in green, respectively.

\section{Conclusions}

A unified controller structure for retarded time-delay systems composed of two different types of free polynomials was derived. An algebraic method for infinite closed-loop spectrum assignment was presented and a search algorithm for the appropriate infinite closed-loop spectrum was proposed. To shape the chains of infinitely many poles, a search criterion based on predetermined regions of poles was established and it was shown that the same technique was applicable for the closed-loop spectrum with uncertain time delays. Finally, optimal robust controller synthesis using mixed sensitivity approach was demonstrated in a control example. 


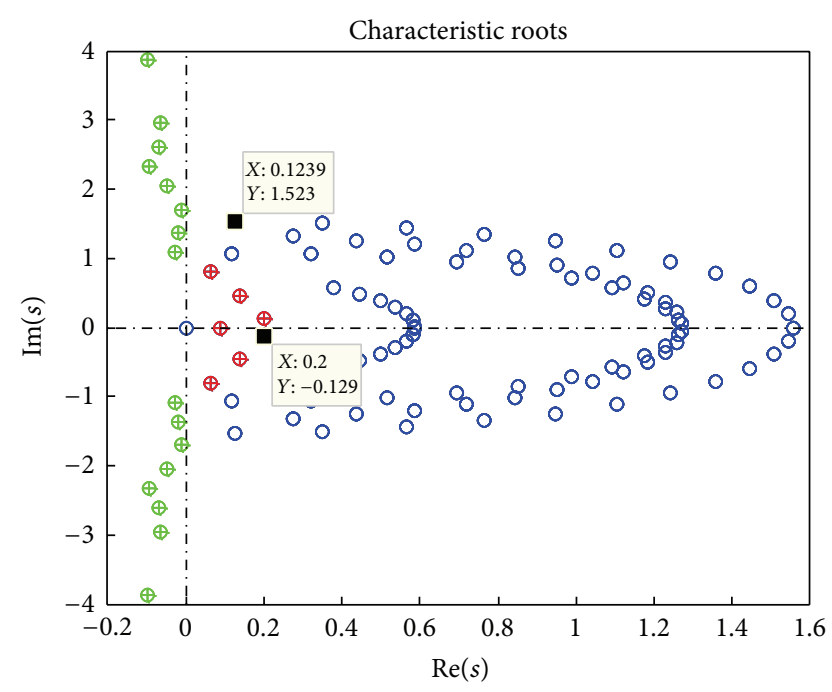

Figure 1: Poles of $\widetilde{T}(s, \lambda)$ shifted by $-r+0.2$ in green and red, respectively; the spectrum $\psi_{0, \bar{\tau}}$ is in blue.

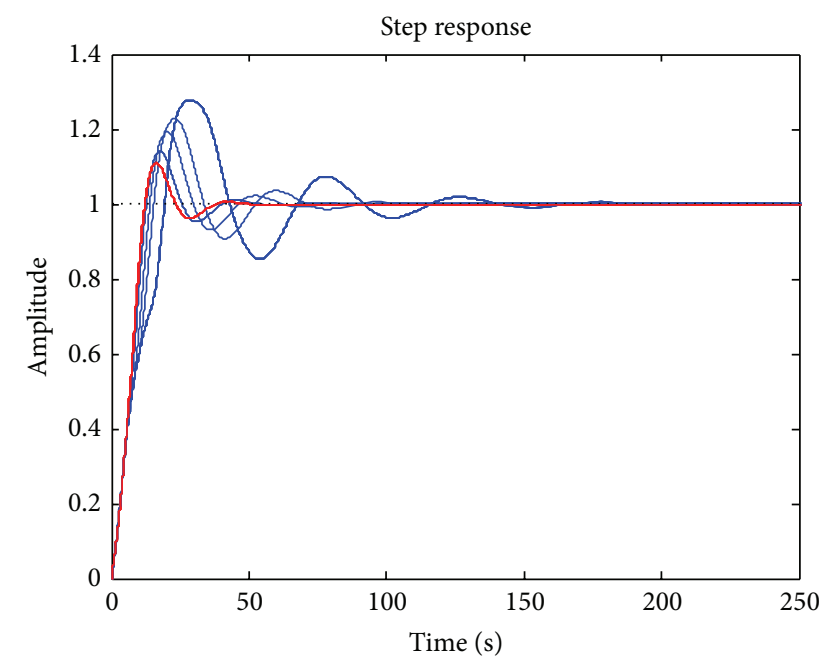

FIGURE 2: Step response of $\widetilde{T}(s, \lambda)$ in blue and of $T(s, \lambda)$ in red.

\section{Appendix}

Proof of Theorem 4. Considering Assumptions 1, 2, and 3, polynomial equations (10) have infinite solutions, where an individual polynomial equation in (10) can be represented in the form of an underdetermined system of linear equations with free parameters. The number of free parameters coincides with the number of parameters in $\kappa_{i}$, which represent an equivalent representation.

As shown in Proposition 2, if in the previous equation free parameters change, this leads to the change of the term on the right side of (10), which results in the change of the solutions in the current equation. Therefore, (10) need to be solved sequentially, but as Theorem 4 , only once. After the first solution of (10), changes of free parameters relative to

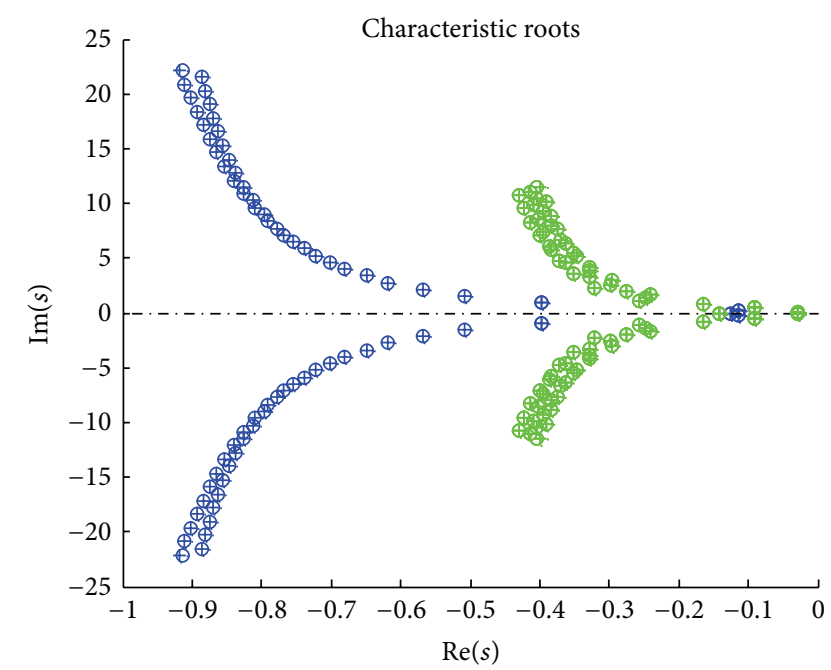

FIgURE 3: Closed-loop poles of $T(s, \lambda)$ in blue and of $\widetilde{T}(s, \lambda)$ in green.

the free parameters of the original solution can be expressed with polynomials $\kappa_{i}$ :

$$
A_{0} R_{N i}+B_{0} P_{N i}+\sum_{j=1}^{i} A_{j} R_{N i-j}+B_{j} P_{N i-j}=D_{i}
$$

Changing the free parameters in the first equation can be represented as a change of controller variables $R_{0}$ and $P_{0}$ to $R_{N 0}=R_{0}-B_{0} \kappa_{0}$ and $P_{N 0}=P_{0}+A_{0} \kappa_{0}$, which results in

$$
A_{0}\left(R_{0}-B_{0} \kappa_{0}\right)+B_{0}\left(P_{0}+A_{0} \kappa_{0}\right)=D_{i}
$$

and the change of the solutions in the second equation

$$
\begin{aligned}
A_{0} R_{N 1}^{\prime}+B_{0} P_{N 1}^{\prime}= & D_{1}-A_{1}\left(R_{0}-B_{0} \kappa_{0}\right) \\
& -B_{1}\left(P_{0}+A_{0} \kappa_{0}\right) .
\end{aligned}
$$

By insertion of $D_{1}$ from (10) into (A.3), the amount of the change of $R_{N 1}^{\prime}$ and $P_{N 1}^{\prime}$ can be expressed in accordance with the change of $R_{N 0}$ and $P_{N 0}$ :

$$
\begin{aligned}
A_{0} R_{N 1}^{\prime} & +B_{0} P_{N 1}^{\prime} \\
& =A_{0} R_{1}+B_{0} P_{1}+A_{1} B_{0} \kappa_{0}-B_{1} A_{0} \kappa_{0} \\
& =A_{0}\left(R_{1}-B_{1} \kappa_{0}\right)+B_{0}\left(P_{1}+A_{1} \kappa_{0}\right) .
\end{aligned}
$$

Considering the change of free parameters in the second equation same as in (A.2), we derive $R_{N 1}=R_{N 1}^{\prime}-B_{0} \kappa_{1}=$ $R_{1}-B_{1} \kappa_{0}-B_{0} \kappa_{1}$ and $P_{N 1}=P_{N 1}^{\prime}+A_{0} \kappa_{1}=P_{1}+A_{1} \kappa_{0}+A_{0} \kappa_{1}$. When substituting $R_{N 1}$ and $P_{N 1}$ in (A.1), the second equation becomes

$$
\begin{gathered}
A_{0}\left(R_{1}-B_{1} \kappa_{0}-B_{0} \kappa_{1}\right)+B_{0}\left(P_{1}+A_{1} \kappa_{0}+A_{0} \kappa_{1}\right) \\
=D_{1}-A_{1}\left(R_{0}-B_{0} \kappa_{0}\right)-B_{1}\left(P_{0}+A_{0} \kappa_{0}\right) .
\end{gathered}
$$




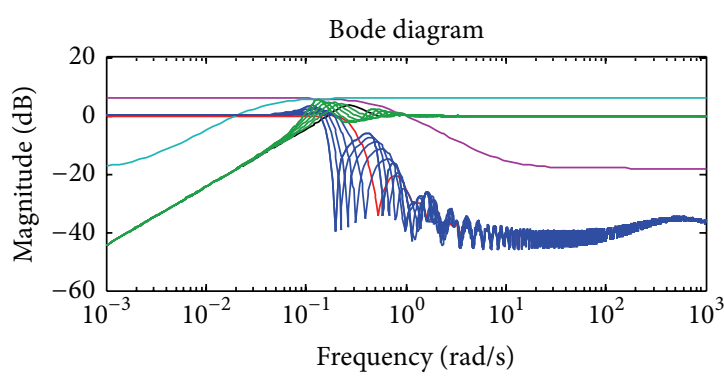

(a)

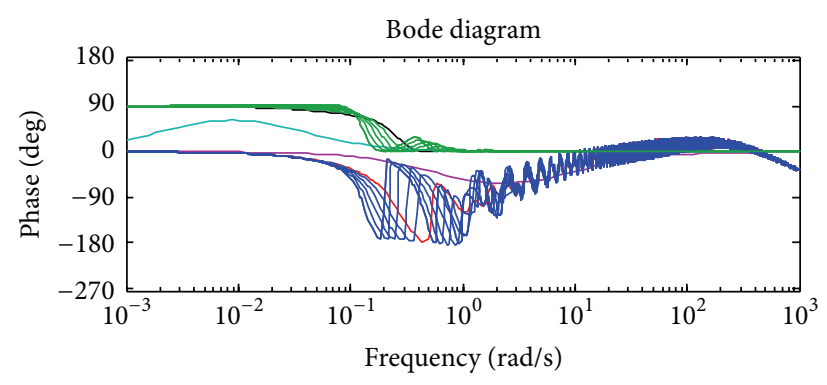

(b)

Figure 4: Bode diagram of $1 / W_{S}$ in pale blue, $1 / W_{T}$ in purple, $T(s, \lambda)$ in red, $\widetilde{T}(s, \lambda)$ in blue, $S(s, \lambda)$ in black, and $\widetilde{S}(s, \lambda)$ in green.

Derivation of appropriate controller variables is executed sequentially. Generally we can write for the $i$ th equation

$$
\begin{aligned}
A_{0} R_{N 1}^{\prime} & +B_{0} P_{N 1}^{\prime} \\
= & D_{i}-\sum_{j=1}^{i} A_{j}\left(R_{i-j}-\sum_{k=0}^{\min \left(i-j, m_{h}\right)} B_{k} \kappa_{i-j-k}\right) \\
& +B_{j}\left(P_{i-j}+\sum_{k=0}^{\min \left(i-j, n_{h}\right)} A_{k} \kappa_{i-j-k}\right) .
\end{aligned}
$$

After replacing $D_{i}$ in (A.6) with an equivalent representation from (10) and cancelation of individual terms, we derive

$$
\begin{aligned}
A_{0} R_{N i}^{\prime}+ & B_{0} P_{N i}^{\prime} \\
= & A_{0} R_{i}+B_{0} P_{i}+\sum_{j=1}^{i}\left(A_{j} R_{i-j}+B_{j} P_{i-j}\right) \\
& -\sum_{j=1}^{i} A_{j}\left(R_{i-j}-\sum_{k=0}^{\min \left(i-j, m_{h}\right)} B_{k} \kappa_{i-j-k}\right) \\
& -B_{j}\left(P_{i-j}+\sum_{k=0}^{\min \left(i-j, n_{h}\right)} A_{k} \kappa_{i-j-k}\right) \\
= & A_{0}\left(R_{i}-\sum_{k=1}^{\min \left(i, m_{h}\right)} B_{k} \kappa_{i-k}\right) \\
& +B_{0}\left(P_{i}+\sum_{k=1}^{\min \left(i, n_{h}\right)} A_{k} \kappa_{i-k}\right) .
\end{aligned}
$$

After considering the influence of free parameters, we derive the final form of $R_{N i}$ and $P_{N i}$ :

$$
\begin{aligned}
A_{0} R_{N i}+B_{0} P_{N i}= & A_{0}\left(R_{i}-\sum_{k=0}^{\min \left(i, m_{h}\right)} B_{k} \kappa_{i-k}\right) \\
& +B_{0}\left(P_{i}+\sum_{k=0}^{\min \left(i, n_{h}\right)} A_{k} \kappa_{i-k}\right) .
\end{aligned}
$$

After replacing controller variables in (A.1) with (A.8), we derive (17), which completes the proof of Theorem 4 .
Proof of Theorem 6. The two presented types of free polynomials expressed in (16) and (19) might be joined in a unified controller structure $K_{\gamma}(20)$. The proof relies on the fact that free polynomials $\lambda$ cancel out in the closed loop (18) and therefore might be included into the controller structure (16) as well. The following closed-loop representation with the controller $K_{\gamma}$,

$$
\begin{aligned}
D= & A\left(R-\sum_{i=0}^{\nu} \sum_{k=0}^{\min \left(i, m_{h}\right)} B_{k} \kappa_{i-k} e^{-i \tau s}-B \lambda\right) \\
& +B\left(P+\sum_{i=0}^{v} \sum_{k=0}^{\min \left(i, n_{h}\right)} A_{k} \kappa_{i-k} e^{-i \tau s}+A \lambda\right),
\end{aligned}
$$

leads to the same closed-loop representation as in (17).

\section{Conflict of Interests}

The authors declare that there is no conflict of interests regarding the publication of this paper.

\section{References}

[1] J. Richard, "Time-delay systems: an overview of some recent advances and open problems," Automatica, vol. 39, no. 10, pp. 1667-1694, 2003.

[2] K. Gu, V. L. Kharitonov, and J. Chen, Stability of Time-Delay Systems, Birkhäuser, Basel, Switzerland, 2003.

[3] M. Wu, Y. He, and J.-H. She, Stability Analysis and Robust Control of Time-Delay Systems, Springer, Beijing, China, 2010.

[4] J. J. Loiseau, W. Michiels, S.-I. Niculescu, and R. Sipahi, Eds., Topics in Time Delay Systems: Analysis, Algorithms and Control, vol. 388 of Lecture Notes in Control and Information Sciences, Springer, Berlin, Germany, 2009.

[5] R. Sipahi, T. Vyhlídal, S.-I. Niculescu, and P. Pepe, Eds., Time Delay Systems: Methods, Applications and New Trends, vol. 423 of Lecture Notes in Control and Information Sciences, Springer, Berlin, Germany, 2012.

[6] Y. Zhang, X. Chen, and R. Lu, "Performance of networked control systems," Mathematical Problems in Engineering, vol. 2013, Article ID 382934, 11 pages, 2013.

[7] Y. Wang, H. R. Karimi, and Z. Xiang, " $H_{\infty}$ control for networked control systems with time delays and packet dropouts," Mathematical Problems in Engineering, vol. 2013, Article ID 635941, 10 pages, 2013. 
[8] W. Michiels, T. Vyhlídal, and P. Zítek, "Control design for timedelay systems based on quasi-direct pole placement," Journal of Process Control, vol. 20, no. 3, pp. 337-343, 2010.

[9] Z. Wu and W. Michiels, "Reliably computing all characteristic roots of delay differential equations in a given right half plane using a spectral method," Journal of Computational and Applied Mathematics, vol. 236, no. 9, pp. 2499-2514, 2012.

[10] K. Engelborghs, T. Luzyanina, and D. Roose, "Numerical bifurcation analysis of delay differential equations using DDEBIFTOOL," ACM Transactions on Mathematical Software, vol. 28, no. 1, pp. 1-21, 2002.

[11] T. Vyhlídal and P. Zítek, "Mapping based algorithm for largescale computation of quasi-polynomial zeros," IEEE Transactions on Automatic Control, vol. 54, no. 1, pp. 171-177, 2009.

[12] D. Breda, S. Maset, and R. Vermiglio, “TRACE-DDE: a tool for robust analysis and characteristic equations for delay differential equations," in Topics in Time Delay Systems, vol. 388 of Lecture Notes in Control and Information Sciences, Springer, Berlin, Germany, 2009.

[13] P. Zítek, "Frequency-domain synthesis of hereditary control systems via anisochronic state space," International Journal of Control, vol. 66, no. 4, pp. 539-556, 1997.

[14] W. Michiels, K. Engelborghs, P. Vansevenant, and D. Roose, "Continuous pole placement for delay equations," Automatica, vol. 38, no. 5, pp. 747-761, 2002.

[15] W. Michiels and T. Vyhlídal, "An eigenvalue based approach for the stabilization of linear time-delay systems of neutral type," Automatica, vol. 41, no. 6, pp. 991-998, 2005.

[16] J. V. Burke, A. S. Lewis, and M. L. Overton, "A robust gradient sampling algorithm for nonsmooth, nonconvex optimization," SIAM Journal on Optimization, vol. 15, no. 3, pp. 751-779, 2005.

[17] J. V. Burke, D. Henrion, A. S. Lewis, and M. L. Overton, "HIFOO-a matlab package for fixedorder controller design and $H_{\infty}$-optimization," in Proceedings of the 5th IFAC Symposium on Robust Control Design (ROCOND '06), pp. 339-344, Toulouse, France, July 2006.

[18] J. Vanbiervliet, K. Verheyden, W. Michiels, and S. Vandewalle, "A nonsmooth optimisation approach for the stabilisation of time-delay systems," ESAIM: Control, Optimisation and Calculus of Variations, vol. 14, no. 3, pp. 478-493, 2008.

[19] J. J. Loiseau, "Algebraic tools for the control and stabilization of time-delay systems," Annual Reviews in Control, vol. 24, pp. 135-149, 2000.

[20] S. Gumussoy, "Coprime-inner/outer factorization of SISO timedelay systems and FIR structure of their optimal $\mathrm{H}$-infinity controllers," International Journal of Robust and Nonlinear Control, vol. 22, no. 9, pp. 981-998, 2012.

[21] M. de la Sen, "On pole-placement controllers for linear timedelay systems with commensurate point delays," Mathematical Problems in Engineering, no. 1, pp. 123-140, 2005.

[22] W. Michiels and S. Niculescu, Stability and Stabilization of Time-Delay Systems. An Eigenvalue Based Approach, SIAM, Philadelphia, Pa, USA, 2007.

[23] J. K. Hale and S. M. Verduyn Lunel, Introduction to Functional Differential Equations, vol. 99 of Applied Mathematical Sciences, Springer, New York, NY, USA, 1993.

[24] J. C. Doyle, B. Francis, and A. Tannenbaum, Feedback Control Theory, Macmillan Publishing, New York, NY, USA, 1990.

[25] A. Howard and R. Chris, Elementary Linear Algebra, John Wiley \& Sons, 10th edition, 2005.
[26] K. Verheyden, Numerical bifurcation analysis of large-scale delay differential equations [Ph.D. thesis], Department of Computer Science, K.U. Leuven, Leuven, Belgium, 2007.

[27] A. Sarjaš, R. Svečko, and A. Chowdhury, "Optimal robust motion controller design using multi-objective genetic algorithm," The Scientific World Journal, vol. 2014, Article ID 978167, 15 pages, 2014.

[28] K. Price, R. Storn, and J. Lampinen, Differential Evolution-A Practical Approach to Global Optimization, Springer, 2005.

[29] K. Zhou, J. Doyle, and K. Glover, Robust and Optimal Control, Prentice-Hall, Englewood Cliffs, NJ, USA, 1995.

[30] P. Albertos and P. García, "Robust control design for long timedelay systems," Journal of Process Control, vol. 19, no. 10, pp. 1640-1648, 2009.

[31] J. E. Normey-Rico and E. F. Camacho, "Unified approach for robust dead-time compensator design," Journal of Process Control, vol. 19, no. 1, pp. 38-47, 2009.

[32] P. Zítek, V. Kučera, and T. Vyhlídal, "Meromorphic observerbased pole assignment in time delay systems," Kybernetika, vol. 44, no. 5, pp. 633-648, 2008.

[33] W. Michiels and D. Roose, "Time-delay compensation in unstable plants using delayed state feedback," in Proceedings of the 40th IEEE Conference on Decision and Control (CDC'01), pp. 1433-1437, Tallahassee, Fla,USA, December 2001.

[34] J. E. Normey-Rico and E. F. Camacho, "Dead-time compensators: a survey," Control Engineering Practice, vol. 16, no. 4, pp. 407-428, 2008. 


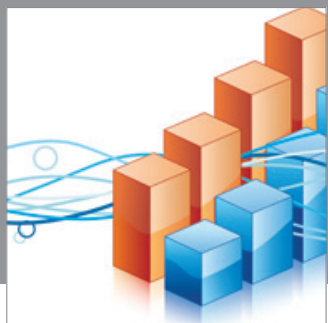

Advances in

Operations Research

mansans

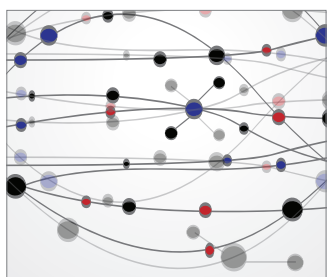

The Scientific World Journal
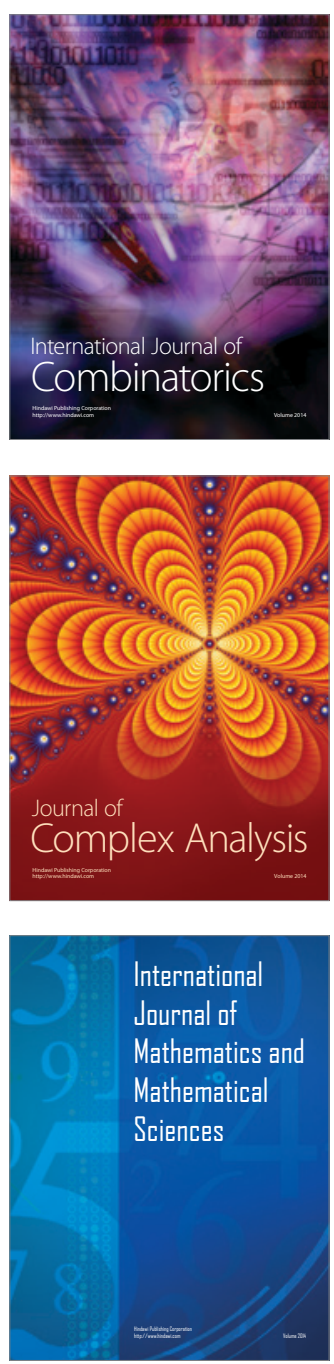
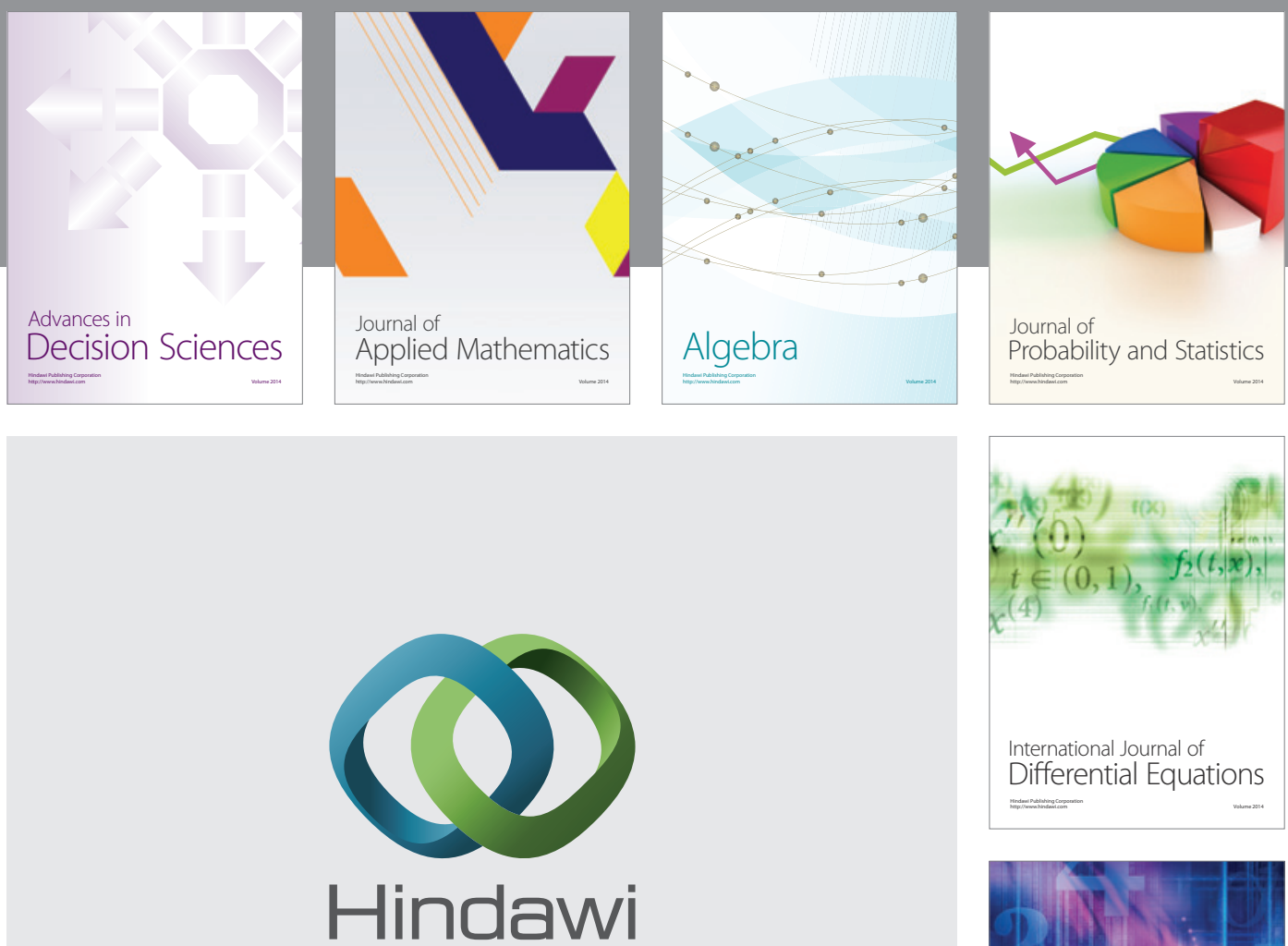

Submit your manuscripts at http://www.hindawi.com
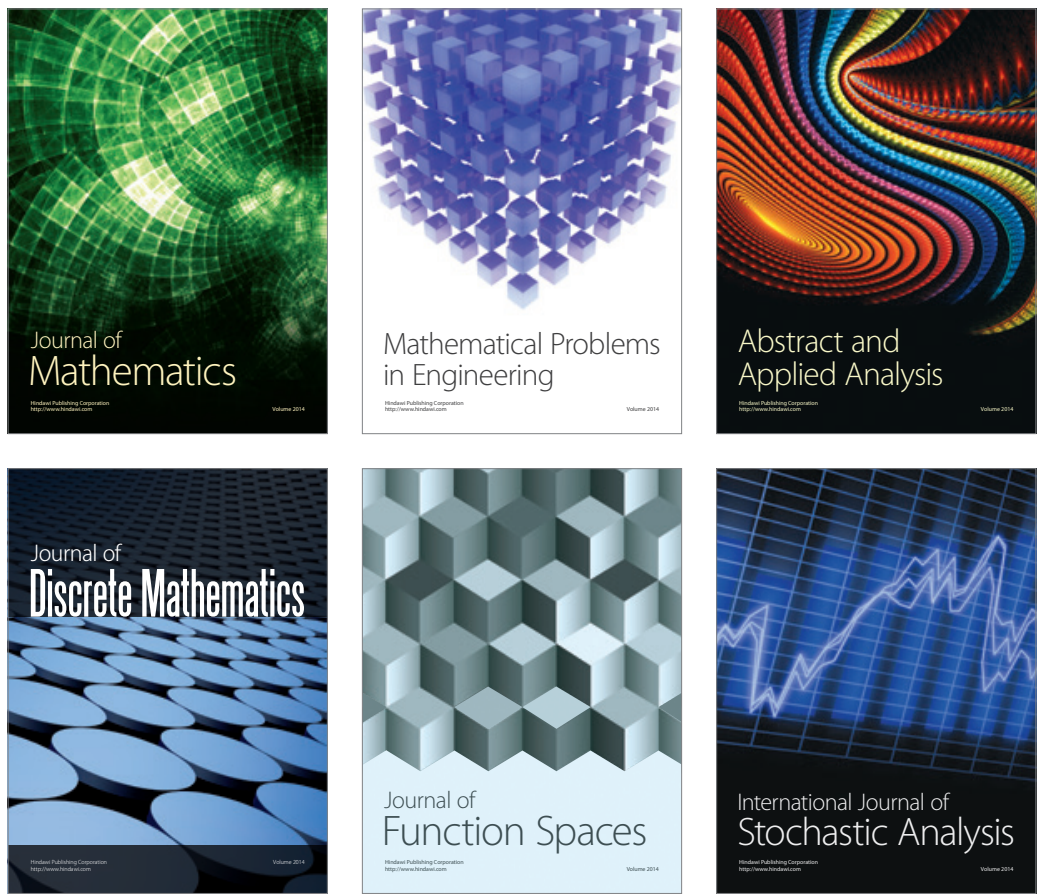

Journal of

Function Spaces

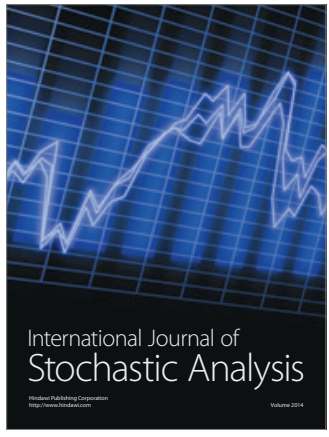

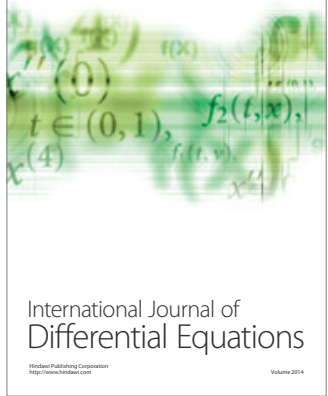
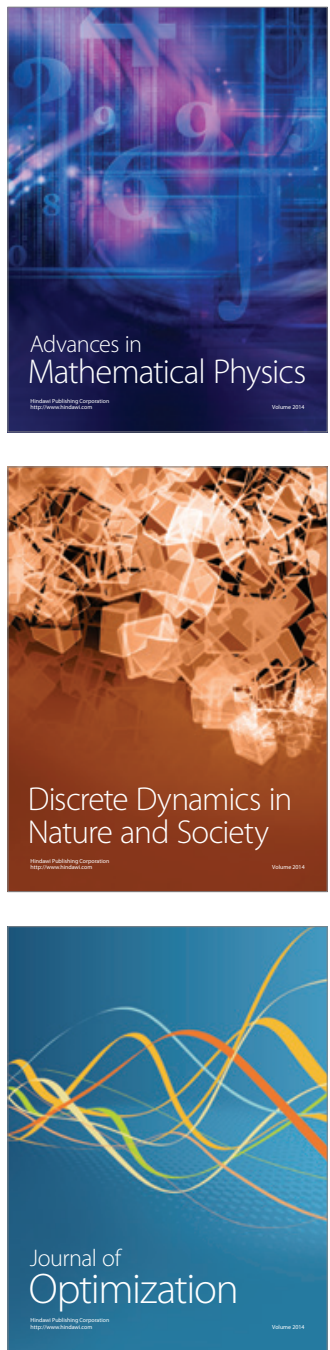